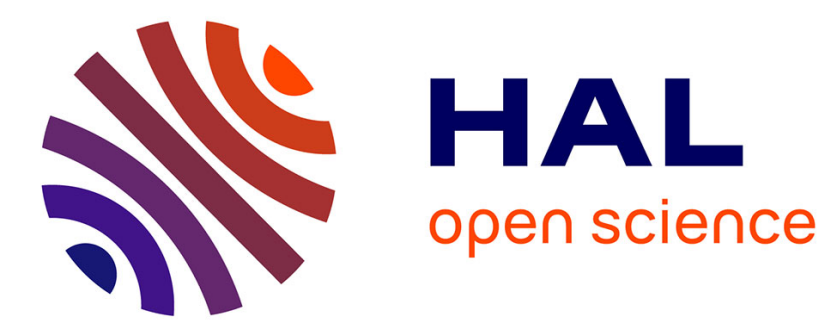

\title{
Contract based Design of Symbolic Controllers for Vehicle Platooning
}

\author{
Adnane Saoud, Antoine Girard, Laurent Fribourg
}

\section{To cite this version:}

Adnane Saoud, Antoine Girard, Laurent Fribourg. Contract based Design of Symbolic Controllers for Vehicle Platooning. 21st International Conference on Hybrid Systems: Computation and Control (part of CPS Week), Apr 2018, Porto, Portugal. pp.277-278, 10.1145/3178126.3187001 . hal-02287002

\section{HAL Id: hal-02287002 https://hal.science/hal-02287002}

Submitted on 13 Sep 2019

HAL is a multi-disciplinary open access archive for the deposit and dissemination of scientific research documents, whether they are published or not. The documents may come from teaching and research institutions in France or abroad, or from public or private research centers.
L'archive ouverte pluridisciplinaire HAL, est destinée au dépôt et à la diffusion de documents scientifiques de niveau recherche, publiés ou non, émanant des établissements d'enseignement et de recherche français ou étrangers, des laboratoires publics ou privés. 


\section{Poster: Contract based Design of Symbolic Controllers for Vehicle Platooning*}

\author{
Adnane Saoud \\ L2S, CNRS \\ CentraleSupélec France \\ adnane.saoud@12s.centralesupelec.fr
}

\author{
Antoine Girard \\ L2S, CNRS \\ CentraleSupélec France \\ antoine.girard@12s.centralesupelec.fr
}

\author{
Laurent Fribourg \\ LSV, CNRS \\ ENS Paris-Saclay France \\ fribourg@lsv.ens-cachan.fr
}

\begin{abstract}
In this work, we present an application of symbolic control and contract based design techniques to vehicle platooning. We use a compositional approach based on continuous-time assume-guarantee contracts. Each vehicle in the platoon is assigned an assumeguarantee contract; and a controller is synthesized using symbolic control to enforce the satisfaction of this contract. The assumeguarantee framework makes it possible to deal with different types of vehicles and asynchronous controllers (i.e controllers with different sampling periods). Numerical results illustrate the effectiveness of the approach.
\end{abstract}

\section{KEYWORDS}

Symbolic control, Contract based design, Vehicle platooning

\section{ACM Reference Format:}

Adnane Saoud, Antoine Girard, and Laurent Fribourg. 2018. Poster: Contract based Design of Symbolic Controllers for Vehicle Platooning. In HSCC '18: 21st International Conference on Hybrid Systems: Computation and Control (part of CPS Week), April 11-13, 2018, Porto, Portugal. , 2 pages. https://doi. org $/ 10.1145 / 3178126.3187001$

\section{INTRODUCTION}

Vehicle platoons are groups of autonomous vehicles traveling closely. Platooning makes it possible to reduce traffic congestion while increasing safety and fuel efficiency. In this paper, we present an approach to vehicle platooning based on the use of symbolic control techniques and assume-guarantee contracts. Due to scalability issues, vehicle platooning can only be solved using compositional techniques. Existing work [1], applies to discrete-time models and specifications and assumes that sampling of time in all components is synchronous. In comparison, our approach makes it possible to take into account the system behavior between sampling instants and to consider asynchronous controllers.

In Section 2, we describe the model of a platoon of vehicles and formulate the control objective. In Section 3, we briefly present

*This work has been supported by Labex DigiCosme (project ANR-11-LABEX-0045DIGICOSME) operated by ANR as part of the program "Investissement d'Avenir" Idex Paris Saclay (ANR-11-IDEX-0003-02).

Permission to make digital or hard copies of part or all of this work for personal or classroom use is granted without fee provided that copies are not made or distributed for profit or commercial advantage and that copies bear this notice and the full citation on the first page. Copyrights for third-party components of this work must be honored

For all other uses, contact the owner/author(s).

HSCC '18, April 11-13, 2018, Porto, Portugal

(C) 2018 Copyright held by the owner/author(s)

ACM ISBN 978-1-4503-5642-8/18/04.

https://doi.org/10.1145/3178126.3187001 the general framework introduced in [3], for compositional reasoning and assign an assume-guarantee contract to each vehicle. In Section 4, we explain how symbolic control techniques can be used to synthesize controllers enforcing the specified contracts. Finally, Section 5 presents numerical simulations of vehicle platooning on circular roads to show the effectiveness of the proposed approach even in the presence of asynchronous sampling and of heterogeneous vehicles in the platoon. (Due to space constraints, the simulations of the vehicle platooning on the straight road are omitted).

\section{PROBLEM FORMULATION}

In this work, we deal with two platoon configurations corresponding to straight and circular roads (see Figure 1). On a straight road, the platoon of $m$ vehicles is made of a leader and $m-1$ followers. The velocity $v_{1}$ of the leader is considered as a disturbance and assumed to belong to the set $\left[0, v_{\max }\right]$ with $v_{\max }>0$. The dynamics of follower $i \in \mathcal{I}=\{2, \ldots, m\}$ is given by the nonlinear system:

$$
\left\{\begin{aligned}
\dot{d}_{i} & =v_{i}-v_{i-1} \\
M \dot{v}_{i} & =F_{i}-f_{0}-f_{1} v_{i}-f_{2} v_{i}^{2}
\end{aligned}\right.
$$

where $d_{i} \leq 0$ represents the relative distance between vehicle $i$ and the preceding vehicle $i-1, v_{i}$ its velocity and $F_{i}$ its control input. On a circular road, there is no leader and the dynamics of each vehicle $i \in \mathcal{I}=\{1, \ldots, m\}$ is given by (1) with the convention that $v_{0}=v_{m}$.

Our control objective is to synthesize controllers, giving values of input $F_{i}$, for all vehicles of a platoon, whatever the number of the vehicles, (except for the leader on a straight road) such that the velocity of each vehicle remains between 0 and $v_{\max }$, and the

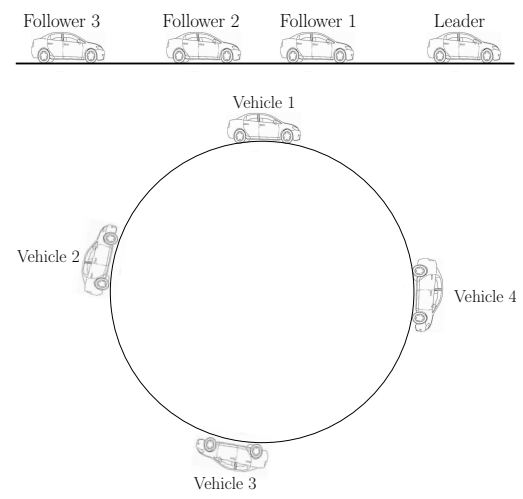

Figure 1: A platoon of 4 vehicles on a straight road (top) or on a circular road (bottom) 
relative distance between two vehicles remains smaller than $d_{\max } \leq$ 0 (i.e. the absolute distance remains larger than $\left.\left|d_{\max }\right|\right)$ :

$$
\forall i \in \mathcal{I}, \forall t \in \mathbb{R}_{0}^{+}, v_{i}(t) \in\left[0, v_{\max }\right] \text { and } d_{i}(t) \in\left(-\infty, d_{\max }\right]
$$

The computational complexity of existing symbolic approaches grows exponentially with the dimension and thus with the number of vehicles in the platoon. A way to tackle this computational complexity is the use of continous-time assume-guarantee contracts.

\section{ASSUME-GUARANTEE CONTRACTS}

A continuous-time system is a tuple $\Sigma=(W, X, Y, \mathcal{T})$ where $W \subseteq$ $\mathbb{R}^{m}, X \subseteq \mathbb{R}^{n}$ and $Y \subseteq \mathbb{R}^{p}$, are the sets of inputs, states, and outputs; and $\mathcal{T}$ is a set of continuous-time trajectories $(w, x, y): \mathbb{R}_{0}^{+} \rightarrow$ $W \times X \times Y$. A continuous-time assume-guarantee contract for $\Sigma$ is a tuple $C=\left(A_{W}, G_{X}, G_{Y}\right)$ where $A_{W} \subseteq W$ is a set of assumptions on the external input and $G_{X} \subseteq X, G_{Y} \subseteq Y$ are sets of guarantees on the state and output, respectively. Intuitively, an assume-guarantee contract is strongly satisfied when if the system's input belongs to $A_{W}$ up to a time instant $t$, then the system's state belongs to $G_{X}$ at least until $t$, and the system's output belongs to $G_{Y}$ until $t+\delta$ with $\delta>0$, see [3].

The dynamics of the platoon on straight or circular roads can be seen as a cascade composition of $m-1$ systems $\Sigma_{2}\left\|_{c} \ldots\right\|_{c} \Sigma_{m}$ or feedback-cascade composition of $m$ systems $\left(\Sigma_{1}\left\|_{c} \ldots\right\|_{c} \Sigma_{m}\right)_{f}$, respectively (see Figure 1). Therefore, in view of Theorems 1 and 2 in [3] , the control objective can be achieved, on both straight and circular roads, by assigning assume-guarantee contracts $C=$ $\left(A_{W}, G_{X}, G_{Y}\right)$ to systems $\Sigma_{i}$ where

$$
A_{W}=G_{Y}=\left[0, v_{\max }\right] \text { and } G_{X}=\left(-\infty, d_{\max }\right] \times\left[0, v_{\max }\right] .
$$

Using the previous results, if one can synthesize a controller for each vehicle in the platoon to enforce the strong satisfaction of the contract (3), then the control objective is achieved. In the following, we provide a solution to the control objective using symbolic control techniques.

\section{SYMBOLIC CONTROLLER SYNTHESIS}

Given a dynamical system described by differential equations, a symbolic model or discrete abstraction is a finite-state dynamical system related to the original system by some formal behavioral relationship (e.g. simulation, bisimulation and their alternating/ approximate versions), which makes it possible to refine a symbolic controller, designed for the abstraction, into a concrete one that can be used on the original system.

For the vehicle platooning problem, we compute a symbolic abstraction for each vehicle under assumptions of the contract, similarly to [2] by discretizing state and input spaces and by computing over-approximations of the reachable sets. Then, we show that the dynamics of the vehicle is related to its abstraction at sampling instants by an alternating simulation relation [4]. Using a fixed-point algorithm, we can synthesize symbolic safety controllers, which after refinement enforce the strong satisfaction of the contract for the original system. Let us remark that even if the symbolic controller acts at discrete sampling instants; the strong satisfaction of the contracts can be certified by construction.
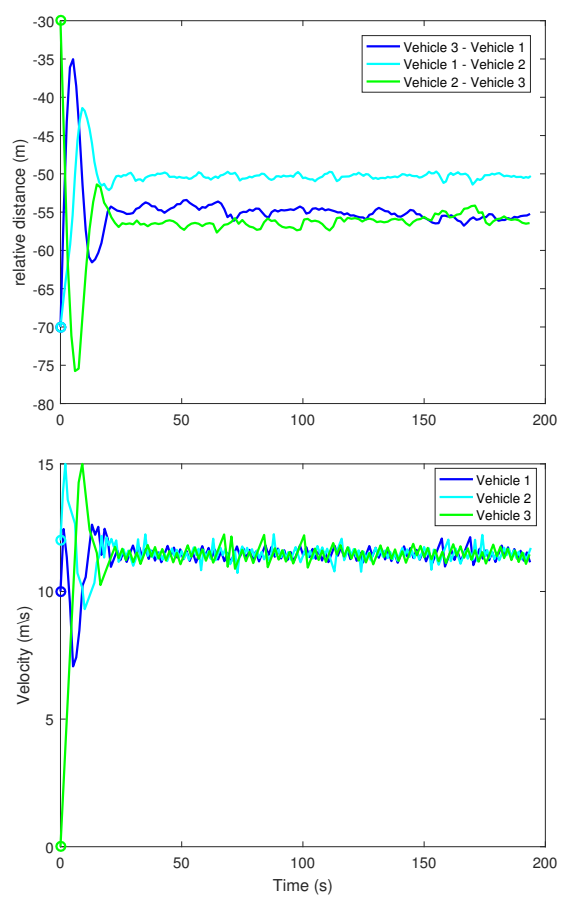

Figure 2: Simulation results for vehicle platooning on a circular road with asynchronous sampling: inter-vehicle distance (top), velocities (bottom).

\section{NUMERICAL RESULTS}

We consider a platoon on a circular road consisting of three vehicles with different sampling periods and different abstraction parameters. Figure 2 shows the simulation results. One can see that the specification is enforced $\left(d_{\max }=-10 \mathrm{~m}\right.$ and $\left.v_{\max }=15 \mathrm{~m} / \mathrm{s}\right)$. Interestingly, the vehicles do not distribute uniformly on the road. On this simulation, one can see that the vehicles with larger sampling period need to keep a larger distance to the front vehicle, which can be explained by the fact, that they need more time to react.

\section{CONCLUSION}

In this paper, we have presented a compositional approach to the control of a platoon of vehicles, based on a notion of continuoustime assume-guarantee contracts. This approach makes it possible to deal with heterogeneous vehicles and asynchronous sampling. In future work, we will deal with additional quantitative objectives (comfort maximization, fuel consumption minimization, etc.).

\section{REFERENCES}

[1] A. Borri, D.V. Dimarogonas, K.H. Johansson, M.D. Di Benedetto, and G. Pola. 2013. Decentralized symbolic control of interconnected systems with application to vehicle platooning. IFAC Proceedings Volumes 46, 27 (2013), 285-292.

[2] P.-J. Meyer, A. Girard, and E. Witrant. 2017. Compositional abstraction and safety synthesis using overlapping symbolic models. IEEE Trans. Automat. Control (2017). To appear.

[3] A. Saoud, A. Girard, and L Fribourg. 2018. On the composition of discrete and continuous-time assume-guarantee contracts for invariance. European Control Conference.

[4] P. Tabuada. 2009. Verification and control of hybrid systems: a symbolic approach. Springer Science \& Business Media. 